\title{
Analysis of Multi-ingredient Food Supplements by Fingerprint HPTLC Approach
}

\author{
Marcello Nicoletti ${ }^{*}$ and Chiara Toniolo \\ Department of Environmental Biology, University Sapienza of Rome, Rome 00185, Italy
}

\begin{abstract}
The increase of import/export of every kind of herbal products calls urgently for adequate controls. Analysis of herbal food supplement (botanicals) is a difficult task, like in the composition determination of a multi-ingredient product, where several botanical drugs were used. Actually, this is an important argument in consideration of health security. The authors reported the results of an analytical approach based on HPTLC (high performance thin layer chromatography) fingerprints comparison and tailored to determine the composition of marketed multi-ingredient botanicals. The method gave positive data in case of the presence of 3-5 species, whereas difficulties were recorded when the number of plants is increased.
\end{abstract}

Key words: Food supplements, multi-ingredient, HPTLC, quality control, fingerprint.

\section{Introduction}

Actually, the quality of food is considered a central problem for human health [1]. Rapid changes in human food environment were caused by rapid urbanization, different lifestyles and longer expectative of live. Our genome is not able to adapt so rapidly, changing only $0.3 \%$ every thousands of years [2]. Among the collateral effects, it is noteworthy the increase of the several pathologies connected with obesity, as well as the appearance of new products properly tailored and marketed to counteract or balance the dysfunctions generated by food changes. The global market of these products is considered relevant and rapidly growing $[3,4]$.

The new products were normally classified in the food supplements sector, but their hybrid nature between drug and food, as well as consumers expectative and request for health positive effects were better expressed by several terms appositively coined, like nutraceuticals [5]. This name is directly derived from the form, recalling that of the pharmaceutical drugs, and their importance in

\footnotetext{
*Corresponding author: Marcello Nicoletti, professor, research fields: phytochemistry, control quality, natural insecticides, and antibiotics. E-mail: marcello.nicoletti@uniroma1.it.
}

consideration of positive benefit in physiological functions and health maintenance. Among nutraceuticals, an important place must be assigned to the food supplements named botanicals, meaning the food supplements containing only, or mainly, plant extracts. Botanicals account for about half of the nutraceuticals market, excluding probiotics.

There is a great debate on nutraceuticals, focused on their claims, utility and security in composition. The last aspect is fundamental and strictly related to quality controls, in particular in botanicals. Botanicals are directly derived from herbal tradition. Uses of herbs in traditional medicines, derived from historical references and empirical evidences, are based on multi-ingredient preparations expressing the in toto activity of the herbal drug mixture. Global market is an easy way for everywhere potential diffusion of traditional herbal drugs in form of botanicals. However, production and marketing are not adequately controlled and increasing import of spiked or adulterated botanicals or herbs generated several alarms and severe consequences [6]. Alarms are real: Medical drugs are subjected to several years of clinical tests, prescription, limit and controls of composition, whereas botanicals are free of controls, 
utilized on the basis of personal advises, but the main problems come from the absence of necessary quality controls. Controls are useful if instrumentation is adequate to the analyzed sample.

Each plant extract contains hundreds of constituents, only considering the detectable ones. Until plants were considered as raw material for obtaining active principles, the control quantity was focused on few substances. Therefore, monographies of herbal drugs in pharmacopoeias are usually based on analysis restricted to one constituent representative of a class of natural products, not considering that similar species contains similar constituents. Furthermore, the botanicals appearance shifted the activity to the phytocomplex and changed completely the analytical point of view and enlarged the perspective to the entire metaboloma.

New instrumentations were properly tailored to face the complexity of plant extracts [7]. HPTLC (high performance thin layer chromatography) is the last evolution of planar chromatography $[8,9]$. HPTLC results in a track of several separated spots, each corresponding to a plant constituent [10]. The total track, named fingerprint, is a reliable imagine of the metabolic production of the analyzed plant or the total extract constitution [11]. The track can be converted in a series of peaks (densitometric conversion) to obtain a quantitative analysis. Identification can be achieved by RF (retention factor) comparison or each spot extracted and analyzed by mass spectrometry, in hyphenated method [12].

\section{Experiments}

\subsection{Materials and Methods}

\subsubsection{Plant Materials}

Extracts utilised as mono-ingredient standards were hydroalcoholic extracts $(10 \mathrm{~g} / 10 \mathrm{~mL})$ obtained from the market or by a lab extraction of identified herbal raw materials, the last also used as reference to confirm the identities of the marketed ones. Detailed information, i.e. producers, production conditions, storage method, etc., can be obtained by directly asking the authors.

\subsubsection{Chromatographic Equipments}

The HPTLC system (CAMAG, Muttenz, Switzerland) consisted of (i) Linomat 5 sample applicator using $100 \mu \mathrm{L}$ syringes and connected to a nitrogen tank; (ii) Chamber ADC 2 containing twin trough chamber $20 \times 10 \mathrm{~cm}$; (iii) Immersion device III; (iv) TLC Plate Heater III; (v) TLC visualizer; (vi) TLC scanner 3 linked to winCATS software. Glass plates $20 \mathrm{~cm} \times 10 \mathrm{~cm}$ (Merck, Darmstadt, Germany) with glass-backed layers silica gel 60 ( $2 \mu \mathrm{m}$ thickness $)$. Before use, plates were prewashed with methanol and dried for $3 \mathrm{~min}$ at $100^{\circ} \mathrm{C}$.

\subsection{Sample Application}

The dried extracts of the analysed samples were weighted and dissolved in methanol $(6 \mathrm{mg} / \mathrm{mL})$. Filtered solutions were applied with nitrogen flow. The operating conditions were: syringe delivery speed,

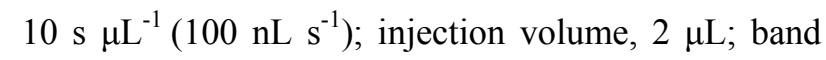
width, $6 \mathrm{~mm}$; distance from bottom, $15 \mathrm{~mm}$.

\subsection{Development and Derivatization}

The HPTLC plates were developed in the automatic and reproducibly developing chamber ADC 2, saturated with the same mobile phase, ethyl acetate: dichloromethane: acetic acid: formic acid: water $(100: 25: 10: 10: 11 ; \quad v / v / v / v)$ for $20 \mathrm{~min}$ at room temperature. The developing solvents (i.e. type of solvents and ratios) were carefully optimized before the analyses. The length of the chromatogram run was $80 \mathrm{~mm}$ from the point of application. The developed layers were allowed to dry in air for $5 \mathrm{~min}$ and then derivatised with a selected solution, including anisaldehyde (1.5 mL p-anisaldehyde, $2.5 \mathrm{~mL} \mathrm{H}_{2} \mathrm{SO}_{4}$, $1 \mathrm{~mL} \mathrm{AcOH}$ in $37 \mathrm{~mL} \mathrm{EtOH}$ ) and/or NPR (natural product reagent) (1 $\mathrm{g}$ diphenylborinic acid aminoethylester in $200 \mathrm{~mL}$ of ethyl acetate), dried in the open air and then dipped into Macrogol reagent (1 $\mathrm{g}$ polyethylene glycol 400 in $20 \mathrm{~mL}$ of 
dichloromethane). Finally, the plates are warmed for 5 min at $120{ }^{\circ} \mathrm{C}$ before inspection. All treated plates were then inspected under a UV (ultra violet) light at $254 \mathrm{~nm}$ or $366 \mathrm{~nm}$ or under reflectance and WRT (white light transmition), respectively, at a Camag TLC visualizer, before and after derivatization. CAMAG DigiStore2 digital system with winCATS software 1.4.3 was used for the documentation of derivatised plates.

Only parts of the images of the HPTLC plates, obtained in different conditions, are reported, because of the available space in this report, but the other images are available under request.

\subsection{Validation}

Sample solutions of the extracts were found to be stable at $4{ }^{\circ} \mathrm{C}$ for at least one month and for at least 3 days on the HPTLC plates. Repeatability was determined by running a minimum of three analyses. $\mathrm{RF}$ values for main selected compounds varied \pm $0.02 \%$. The effects of small changes in the mobile phase composition, mobile phase volume, duration of saturation were minute and reduced by the direct comparison. On the contrary, the results were critically dependent on pre-washing of HPTLC plates with methanol.

\section{Results and Discussion}

If the analysis of a single extract is a difficult job, the situation is very complicated when botanicals are derived from the utilization of several plant extracts. Botanicals are more or less directly derived from the tradition and rituals of millenary traditional medicine. Herbalist knows personally plants to be used and the suitable preparations. On the contrary botanicals are technological products derived from a sequence of industrial steps, including the market of processed raw materials very distant from the geographic and form origins. So far, solutions for this kind of products were essentially based on identification of the main species by marker compounds. Analysis of multi-ingredient botanicals reported the use of NMR (nuclear magnetic resonance) or HPLC (high performance liquid chromatography) by comparison with single selected markers, like ginsenosides to test the presence of Gingko biloba [13].

The importance of HPTLC tool is based on the efficacy in separation and consequently the possibility in checking the entire metaboloma and easily evidencing similarities and differences [14]. Albeit HPLC remains the best analytic tool, HPTLC is practical, low cost, easy to perform, time and solvent saving. Furthermore, the results are very evident and can be understand also by people which have no chemistry knowledge.

The method here described is based on fingerprint approach. WHO, US Herbal Pharmacopoeia and P.R. Chinese Pharmacopoeia confirmed utility and importance of HPTLC and fingerprint approach [15-18]. Considering that an extract can be identified on the basis of its fingerprint, the authors obtained the fingerprint of each species reported in the label, as individual track of the corresponding extract. The fingerprint of the mixture of the extracts was also obtained. All tracks, including that of the mixture, were compared with that of the analyzed product. In principle, a part from quantitative differences, the fingerprint of the mixture made in laboratory and that of the product should be very similar.

In Figs. 1-3, the authors report the results obtained applying the proposed method to three different marketed botanicals. In Fig. 1, the simplest case of a botanical containing two species is reported. The presence of predominant spots in the fingerprint is easy to follow in each track. As expected, the track of the mixture is fixing with that of the analysed botanical. In Fig. 2, a similar product is reported, but the second species is present with two different plant drugs, i.e. flowers and fruits, in order to show the efficiency of the method in such case. Finally, in Fig. 3 , the analysis of a botanical made by three different species is reported, showing the evidence of the 


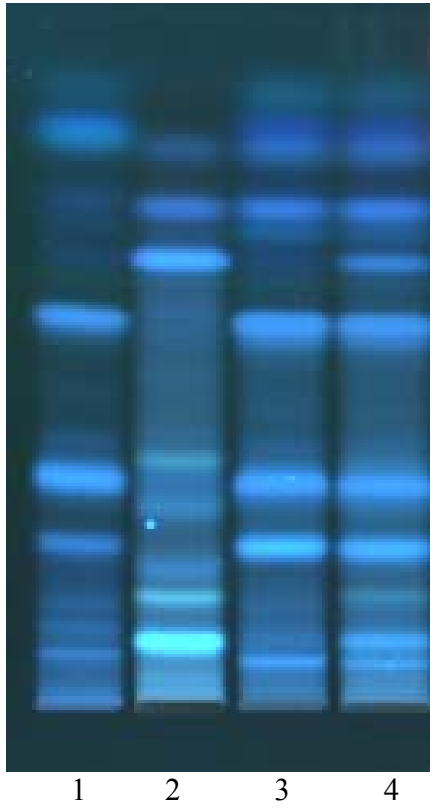

(a)

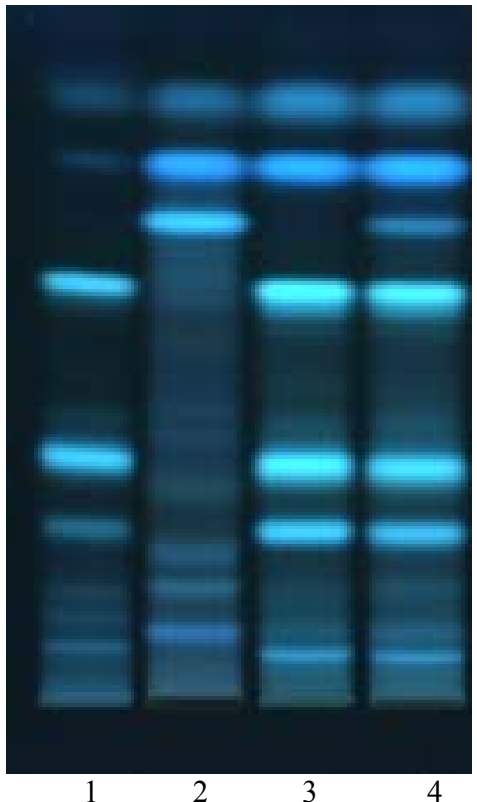

(b)

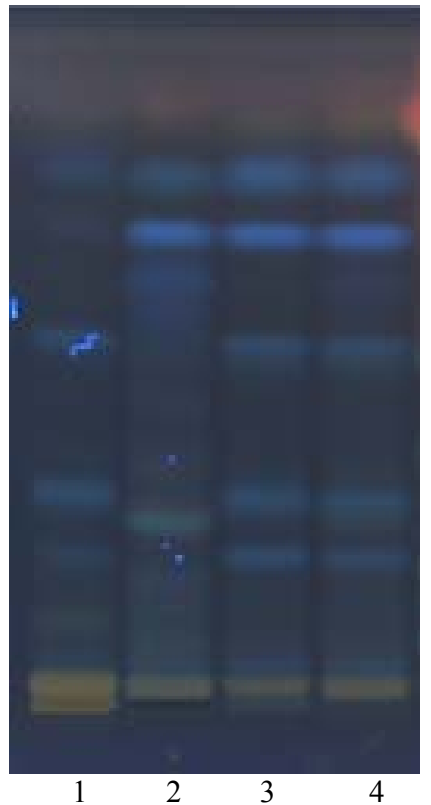

(c)

Fig. 1 HPTLC analysis on a multiingredient botanical containg lemon balm (Melissa officinalis L.) and greater burdock (Arctium lappa L.). (a): visualization: $366 \mathrm{~nm}$; derivatization: none. (b): visualization: $366 \mathrm{~nm}$; derivatization: NP reagent; (c): visualization: $366 \mathrm{~nm}$; derivatization: NP reagent and Anhysaldeide. Tracks: 1, marketed analysed product; 2, lemon balm extract standard fingerprint; 3, greater burdock extract standard fingerprint; 4, mixture of extracts of tracks 2 and 3.

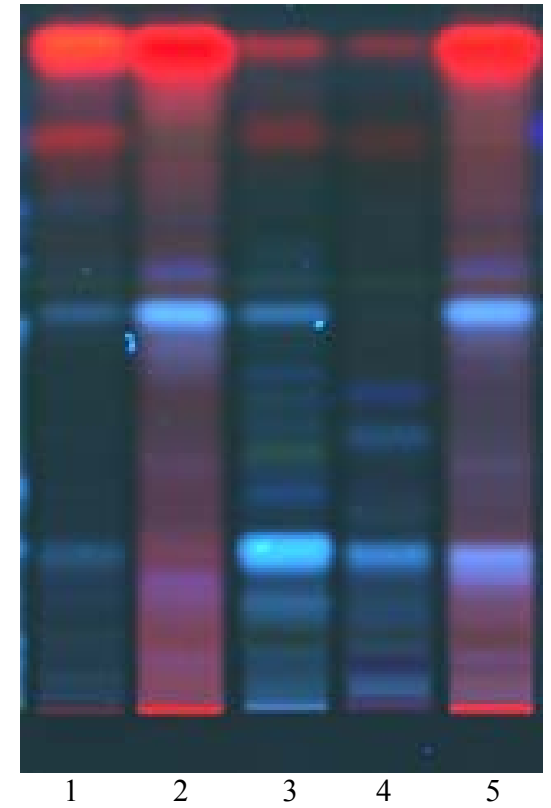

(a)

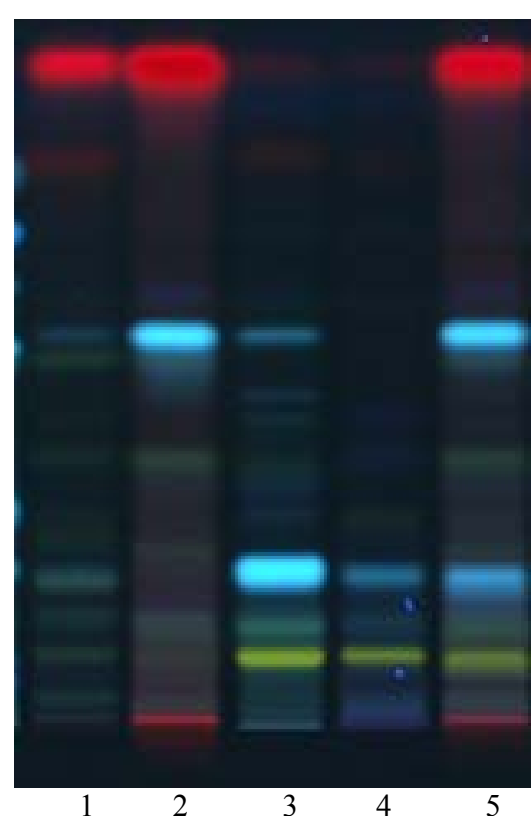

(b)

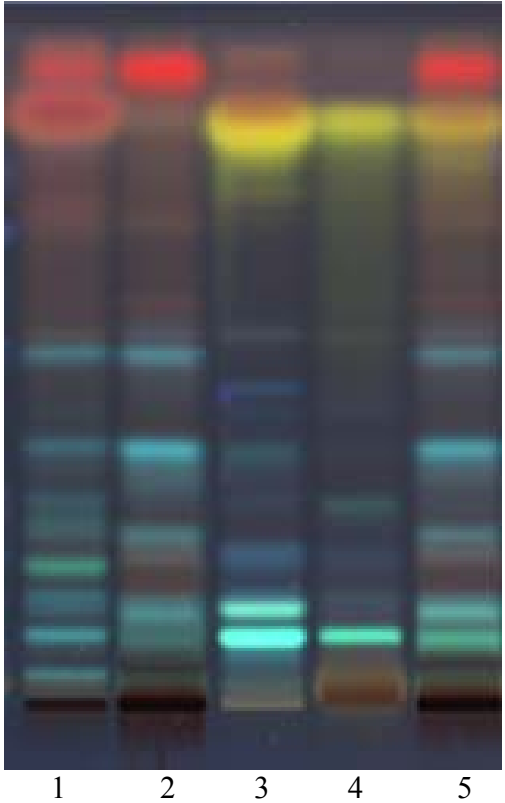

(c)

Fig. 2 HPTLC analysis on a multiingredient botanical containg common horsetail (Equisetum arvense L.) and common elder (Sambucus nigra L.). (a): visualization: $366 \mathrm{~nm}$; derivatization: none. (b): visualization: $366 \mathrm{~nm}$; derivatization: NP reagent; (c): visualization: $366 \mathrm{~nm}$; derivatization: NP reagent and Anhysaldeide. Tracks: 1, marketed analysed product; 2, common horsetail extract standard fingerprint; 3, common elder flowers extract standard fingerprint; 4, common elder flowers extract fruits standard fingerprint; 5, mixture of extracts of tracks 2-4. 


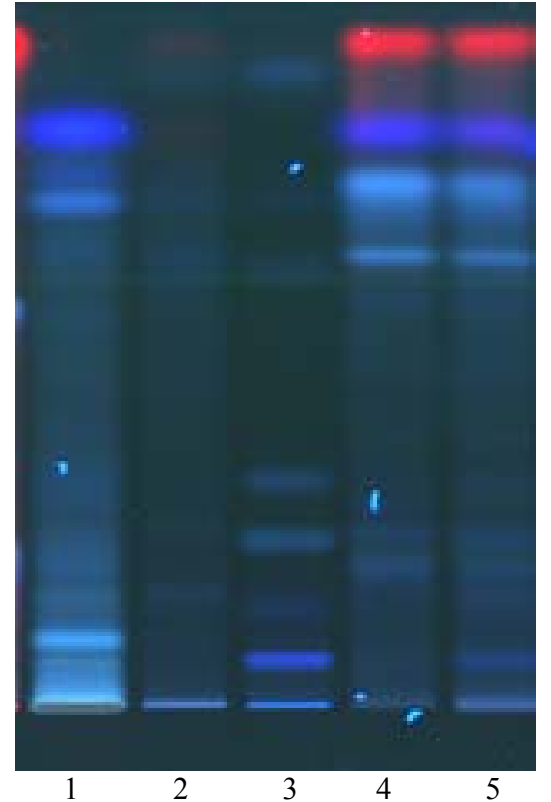

(a)

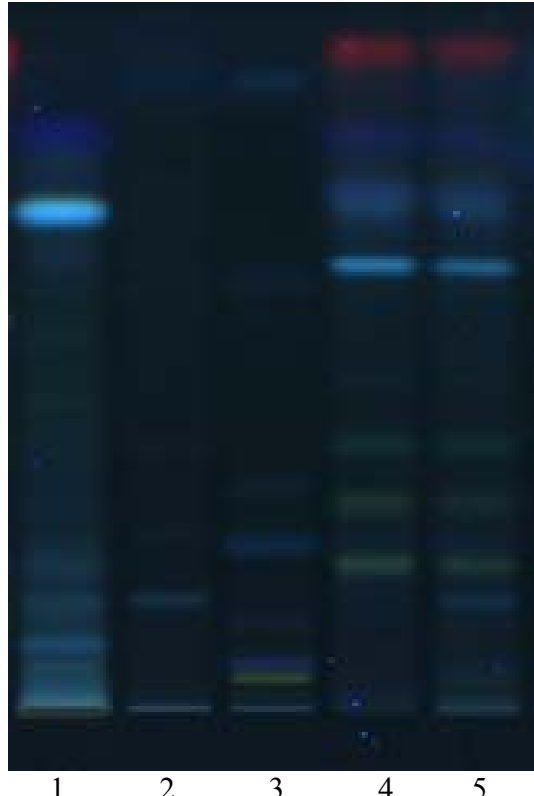

(b)

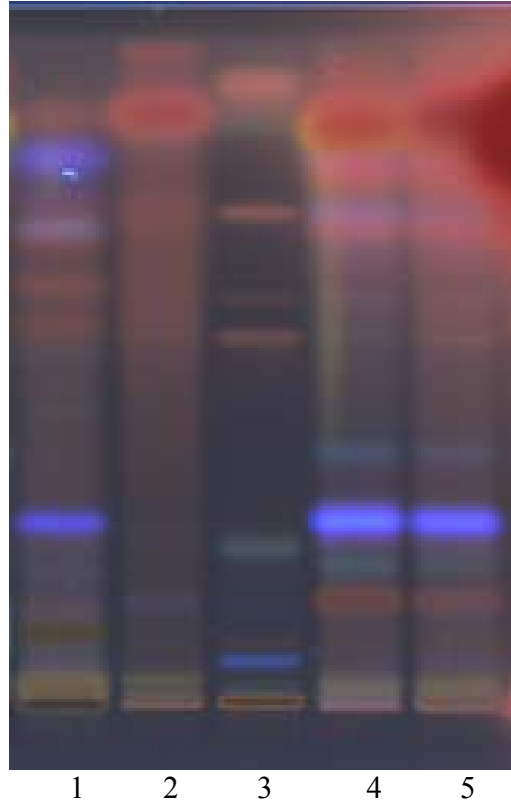

(c)

Fig. 3 HPTLC analysis on a multiingredient botanical containg common horsetail (Equisetum arvense L.) and common elder (Sambucus nigra L.). (a): visualization: $366 \mathrm{~nm}$; derivatization: none. (b): visualization: $366 \mathrm{~nm}$; derivatization: NP reagent; (c): visualization: $366 \mathrm{~nm}$; derivatization: NP reagent and Anhysaldeide. Tracks: 1, marketed analysed product; 2, bay tree (Laurus nobilis L.) extract standard fingerprint; 3, marshmallow (Althea officinalis L.); 4, English lavander (Lavandula angustifolia $L_{\text {.) }}$ extract standard fingerprint; 5, mixture of extracts of tracks 2-4.

reliability of the method also in this case. However, further analysis showed that with the increasing of the number of herbal species present in the product, the identification of all the drugs resulted quite difficult, because of the quantity of spots and the overlapping in some zones of the track. Therefore, each case must be individually considered in its complexity and the method consequently adapted, including the use of selected markers in order to help the identification of some species.

\section{Conclusions}

Analysis of multi-ingredient botanical food supplements is still a problem. In the analyses the fingerprint of the marketed multi-ingredient botanicals was compared with the fingerprints of the extracts of the constituent plants and a full mixture of the constituent extracts in a ratio as possible similar to that of the marketed product, when reported.

In concern with the fingerprint method the authors proposed a different approach. The method appeared useful and reliable for mixture of 3-5 species, whereas results are not totally reliable as the number of species increases. In these cases the identity of some species can still be achieved but not the totality of the composition owing to the quantity of different constituents present. As a matter of fact plants present in low quantity can not be certainly identified and possible overlapping of spots can complicate the situation.

However, the approach resulted positive, rapid and low cost, in particular adapt to evidence the presence of herbs, or other constituents, not reported in the label. In other cases, a supplement of analysis is required.

\section{References}

[1] Jimenez-Chillaron, J. C., and Diaz, R. 2012. "The Role of Nutrition on Epigenetic Modification and Their Implication on Health." Biochimie 94: 2242-63.

[2] Constantin, N., and Wahli, W. 2013. "Nutrigenomic Foods." Nutrafoods 12: 3-12.

[3] Sumeet, K., and Das Madhusweeta. 2011. "Functional Food: An Overview." Food Sci. Biothechnol 20 (4): 
861-75.

[4] Nicoletti, M. 2012. "Nutraceuticals and Botanicals: Overview and Perspectives." International Journal of Food Sciences and Nutrition 63 (S1): 2-6.

[5] De Felice, L. S. 1995. "The Nutraceutical Revolution: Its Impact on Food Industry R\&D.” Trends in Food Science \& Technology 6 (2): 59-61.

[6] Nicoletti, M. 2011. "HPTLC Fingerprint: A Modern Approach for the Analytical Determination of Botanicals." Rev. Bras. Farmacogn 21 (5): 818-23.

[7] Nicoletti, M. 2012. "HPTLC Nutraceuticals and Botanicals Appeal for New Analytical Solutions." Journal Chromatographic Separation Techniques 3: 5.

[8] Zlatkis, A., and Kaiser, R. E. 1977. "HPTLC High Performance Thin Layer Chromatography." Journal of Chromatography Library 9. N.Y.: Elsevier Scientific Publishing.

[9] Reich, E. and Schibli, A. 2006. High-Performance Thin-Layer Chromatography for the Analysis of Medicinal Plants. N.Y.: Thieme Medical Pub.

[10] Gallo, F. R., Multari, G., Federici E., Palazzino, G., Nicoletti, M., and Petitto, V. 2012. "The Modern Analytical Determination of Botanicals and Similar Novel Natural Products by the HPTLC Fingerprint Approach." Studies in Natural Products Chemistry 37: 217-58.

[11] Gallo, F. R., Multari, G., Federici, E., Palazzino, G., and
Nicoletti, M. 2013. "Bearberry Identification by a Multidisciplinary Study on Commercial Raw Material." Natural Products Research 27 (8): 735-42.

[12] Moricz, A. 2014. HPTLC-Bioassay-MS, A Rapid Tool to Search and Analyse Bioactive Plant Products. International Symposium for High-Performance Thin-Layer Chromatography. Lyon 2-4.

[13] Zheng, X. X., Tang, A. Q., Lu, P., Chen, H., Wu, J., and Tang, D. Q. 2013. "Quality Assessment of Ginkgo Biloba Extract from Different Manufacturers of Xuzhou City (China) by HPLC-DAD." Lat. Am. J. Pharm. 32 (2): 175-83.

[14] Toniolo, C., Nicoletti, M., Maggi, F., and Venditti, A. 2013. "Determination by HPTLC of Chemical Composition Variability in Raw Materials Used in Botanicals." Natural Product Research 10: 231-6.

[15] AHP. 2003. American Herbal Pharmacopoeia and Therapeutic Compendium. Scotts Valley, CA.

[16] AOAC. 2005. Official Methods of analysis of AOAC International, 18th Ed. AOAC International, Gaithersburg, MD.

[17] Chinese Pharmacopoeia. 2009. TLC Atlas of Chinese Crude Drugs in Pharmacopoeia of the People's Republic of China Commission. Beijing: People's Medical Publishing House.

[18] WHO. 2000. General Guidelines for Methodologies on Research and Evaluation of Traditional Medicine. World Health Organization, Geneva. 